This item was submitted to Loughborough's Research Repository by the author.

Items in Figshare are protected by copyright, with all rights reserved, unless otherwise indicated.

\title{
Solder paste reflow modeling for flip chip assembly
}

PLEASE CITE THE PUBLISHED VERSION

PUBLISHER

(C) IEEE

\section{LICENCE}

CC BY-NC-ND 4.0

\section{REPOSITORY RECORD}

Mannan, Samjid H., D. Wheeler, David A. Hutt, David C. Whalley, Paul P. Conway, and Chris Bailey. 2019. "Solder Paste Reflow Modeling for Flip Chip Assembly". figshare. https://hdl.handle.net/2134/3926. 
This item was submitted to Loughborough's Institutional Repository (https://dspace.lboro.ac.uk/) by the author and is made available under the following Creative Commons Licence conditions.

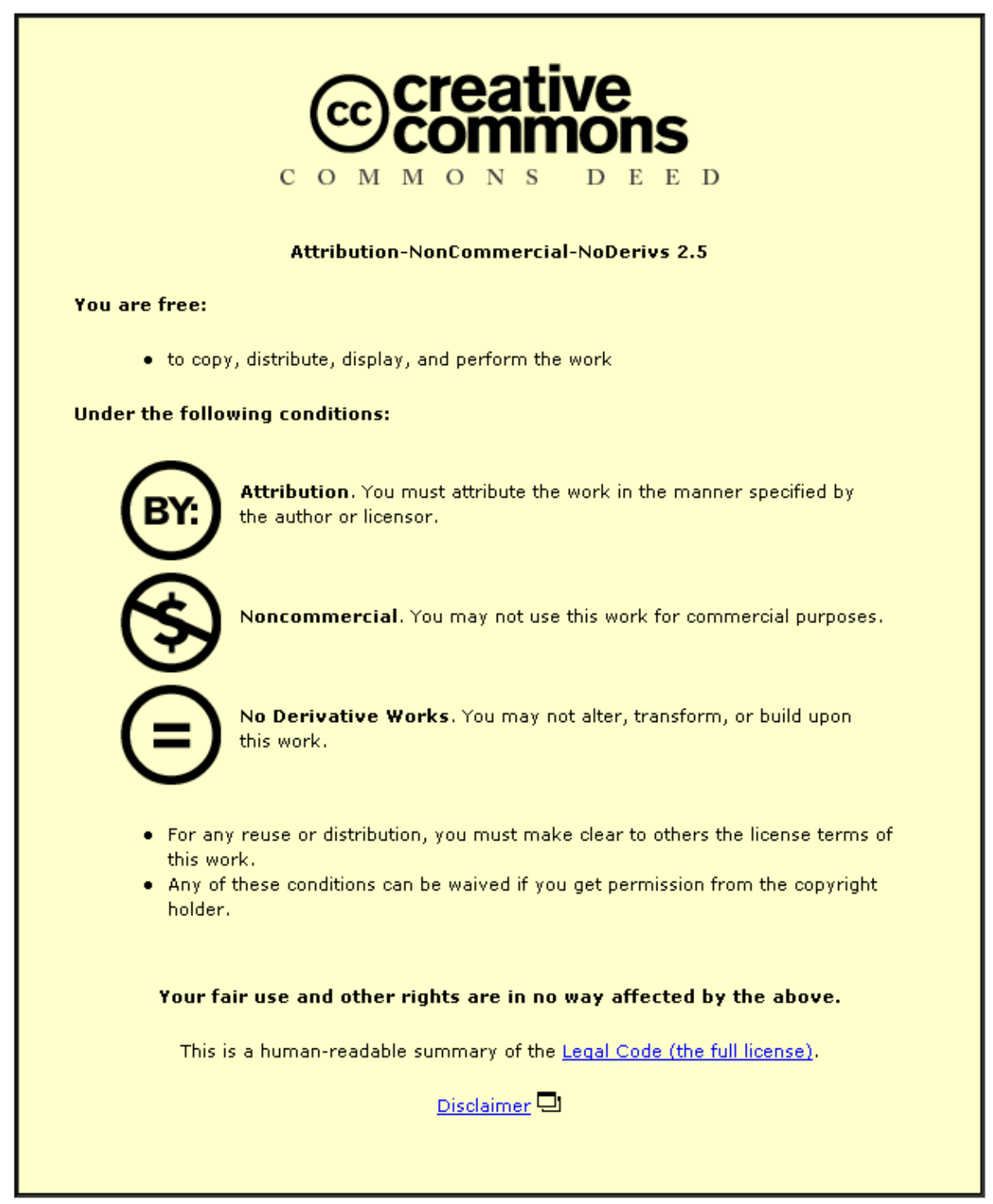

For the full text of this licence, please go to: http://creativecommons.org/licenses/by-nc-nd/2.5/ 


\title{
Solder Paste Reflow Modeling for Flip Chip Assembly
}

\author{
S H Mannan ${ }^{1}$, D Wheeler ${ }^{2}$, D A Hutt ${ }^{3}$, D C Whalley ${ }^{3}$, P P Conway ${ }^{3}$, C Bailey ${ }^{2}$ \\ King's College London ${ }^{1}$, Dept. of Mechanical Engineering, Strand, London WC2R 2LS, UK. \\ Greenwich University ${ }^{2}$, School of Computing and Mathematical Sciences, London, UK. \\ Loughborough University ${ }^{3}$, Dept. of Manufacturing Engineering, LE11 3TU, UK. \\ Tel $^{1}$ : +44 (0)20 7848 1780, Fax $^{1}:$ +44 (0)20 7848 2932, email ${ }^{1}$ : samjid.mannan@kcl.ac.uk
}

\begin{abstract}
Solder paste printing and reflow can provide low cost techniques producing the solder bumps on flip chips. Solder paste consists of a dense suspension of solder particles in a fluid medium (vehicle) that acts as an oxide reducing agent (flux) during reflow, cleaning the metal surfaces of oxides. This paper reports on optical observations of paste behaviour at the small length scales associated with flip chip solder joints, and attempts to model the process using Computational Fluid Dynamics (CFD). Comparison of optical observations and CFD modelling show that the behaviour of the solder cannot be described simply by surface tension and viscous flow effects and it is deduced that oxides are still present on the solder surfaces during the early stages of reflow. The implications for paste heating method and solder volume are discussed, and a preliminary CFD model (based on FIDAP) incorporating the effect of the oxide layers is presented.
\end{abstract}

\section{Introduction}

Solder paste printing and reflow are standard processes used during Surface Mount Assembly. Solder paste reflow involves the coalescence of solder spheres which are typically 5-25 microns or smaller for flip chip applications, into a single solder mass which wets the surfaces to be soldered and then solidifies to form a soldered joint. Before the solder spheres can merge and wet solderable surfaces, oxide layers on the balls and the solderable surfaces must be removed. If the oxide layers are not completely removed then the spheres will only partially merge, or not at all. Due to a continuous process of electronics miniaturisation, the solder joints for flip chip devices have become smaller in volume, leading to smaller particle sized pastes for ease of deposition. This, together with the smaller number of solder particles required to make a joint, can lead to incomplete reflow of the solder particles and solder balling.

This paper reports on a series of optical and CFD studies designed to increase our understanding of the reflow process. The methods used complement previous studies of solder paste dynamics using optical methods but at lower magnification, [1] and also Environmental Scanning Electron Microscopy (ESEM) studies of the reflow process. [2] While CFD studies of void formation and circulation have been carried out previously, this is the first attempt at modelling the coalescence of discrete solder particles known to the authors.

Optical observations of solder paste reflow at high magnification are reported first, describing the preliminary growth of small necks between particles, followed by a faster coalescence process as the particles are pulled into a single central mass. These observations are then correlated with 2$\mathrm{D}$ and 3-D CFD simulations, and it is deduced that the oxide layers produce a significant restraining force on the particles. This is confirmed by observing the effects of paste volume and reflow in Nitrogen or air atmospheres. Finally a CFD model incorporating the effects of an initial oxide layer, fluxoxide reactions and metal re-oxidation reactions is presented. The model is not yet at the stage when quantitative predictions may be made but illustrates some of the phenomena that are observed.

\section{Video Observations}

Video observations have been conducted with the Hirox $^{\mathrm{TM}}$ video system which combines a high magnification zoom lens system with a good focal depth and $\sim 10 \mathrm{~mm}$ working distance from the lens. It is necessary to have a few mm clearance between solder and lens because of the heat involved and the flux fumes from the paste.

Two basic types of observations were made; in the first type, solder was sandwiched between glass plates at a fixed separation. In the second type of experiment, solder paste was placed on a substrate and viewed without constrictions. In both cases the paste was placed on a hotplate and heated while observed from above. The advantage of the first type is that the particles remain a fixed distance from the lens, but the disadvantage is that trapped volatiles form bubbles that can obscure the process. Coalescence of particles in typical flip chip application solder pastes, as well as 650 micron diameter solder particles coated thinly with flux were observed and both processes were also modelled using CFD. The results proved conclusively that the oxide layers on the surface of the solder have not been completely removed, especially during the early stages of coalescence.

The video observations show that the inner layers of the deposit coalesce first, pulling in the outer particles as they merge. This is shown in fig. 1 where a central solder mass is forming. 
Figure 1. Photograph of solder sphere with partially reflowed particles (viewed from below glass substrate)

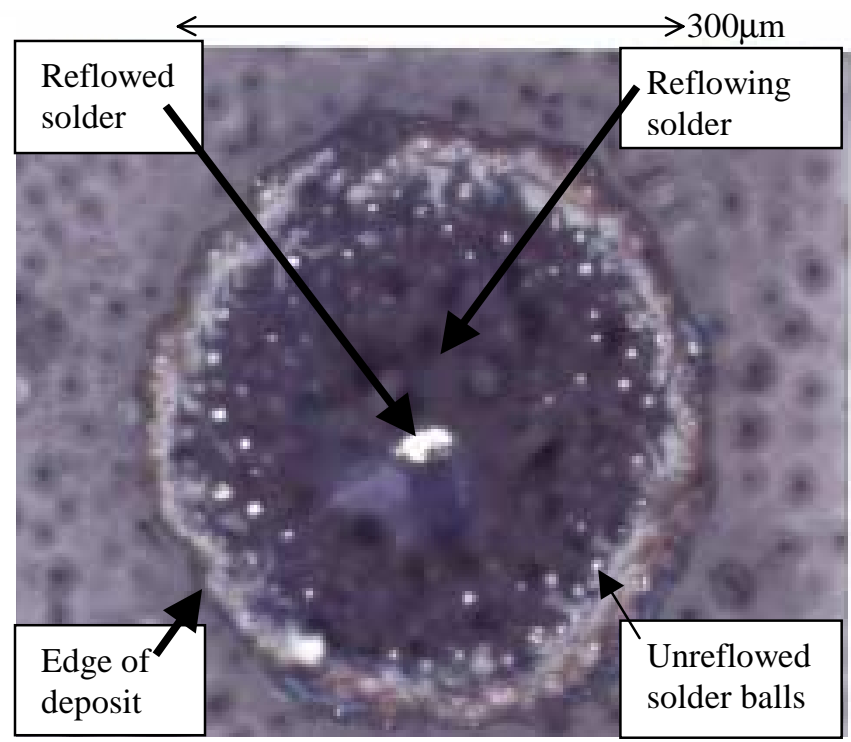

In deposits where reflow is incomplete, the outer particles are often seen partially submerged in the solder mass, but with the top hemispheres retaining their original shape due to oxide layers as sketched in fig. 2 .

\section{Figure 2. Incomplete Coalescence}

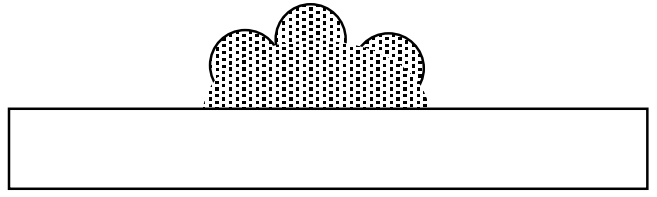

It is assumed that the reason that the inner layers coalesce first is the slight temperature difference that exists between inner and outer layers. Another possibility is that the oxides on the inner particles are reduced more rapidly than on the outer particles. When the heat is supplied from the surrounding atmosphere, as in convection reflow ovens, it produces a more even pattern of coalescence throughout the paste as seen in fig.3, supporting the temperature difference hypothesis.

\section{Figure 3. Hot air heating (viewed from below)}

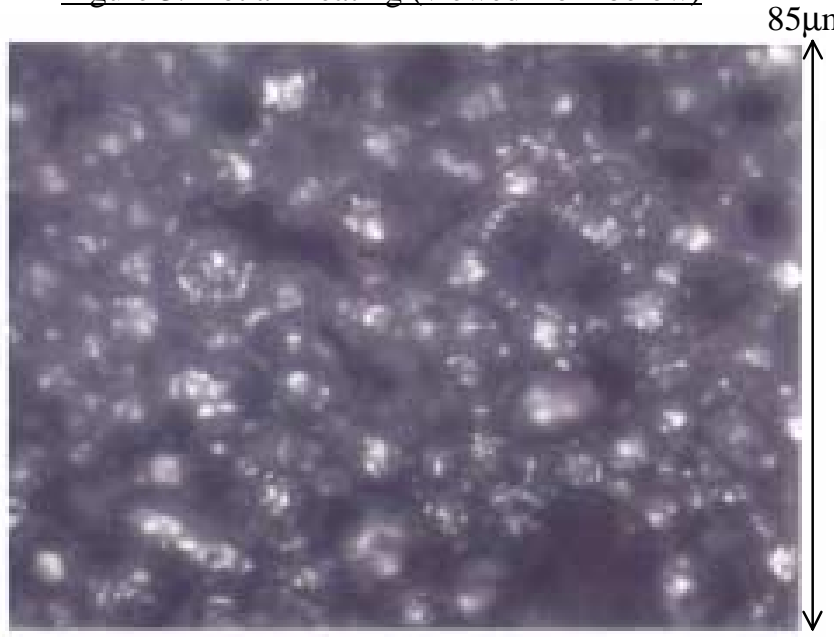

Figure 4. Stages in particle coalescence

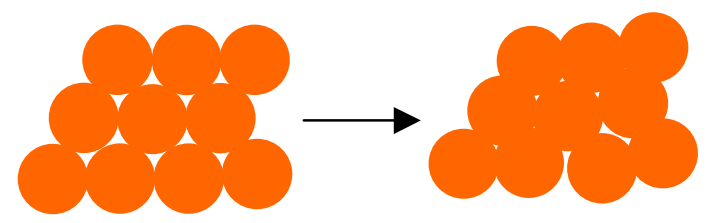

Solvent evaporation pulls Necks form as solder melts particles into contact

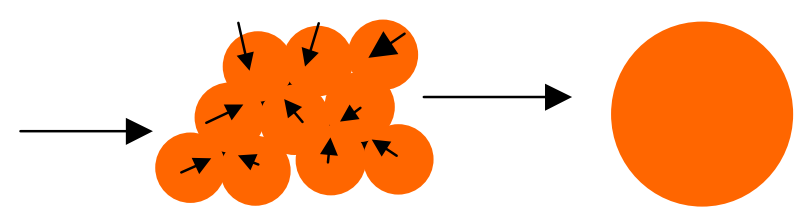

Gaps fill with solder

Rapid movement

Figure 4 shows the main stages observed during solder paste reflow. Once the reflow temperature has been reached, the solder particles initiate necks with their nearest neighbours; typically 2-5 necks per particle. Then these necks enlarge, and the gap between particles is filled either by a central solder mass wicking up between the particles, or by the spheres surrounding a gap moving fractionally closer together. Occasional isolated particles at the periphery can either be rapidly absorbed by the central mass, or remain isolated (fig.5 shows an extreme case of this, created by heating from below with a very slow ramp rate), or coalesce with other particles at the periphery; solder balling. In the later stages of reflow, coalescing particles take fractions of a second to coalesce while at the early stages the oxide layers limit coalescence times to seconds.

Figure 5. Photograph of solder spheres with ring of isolated particles (viewed from below)

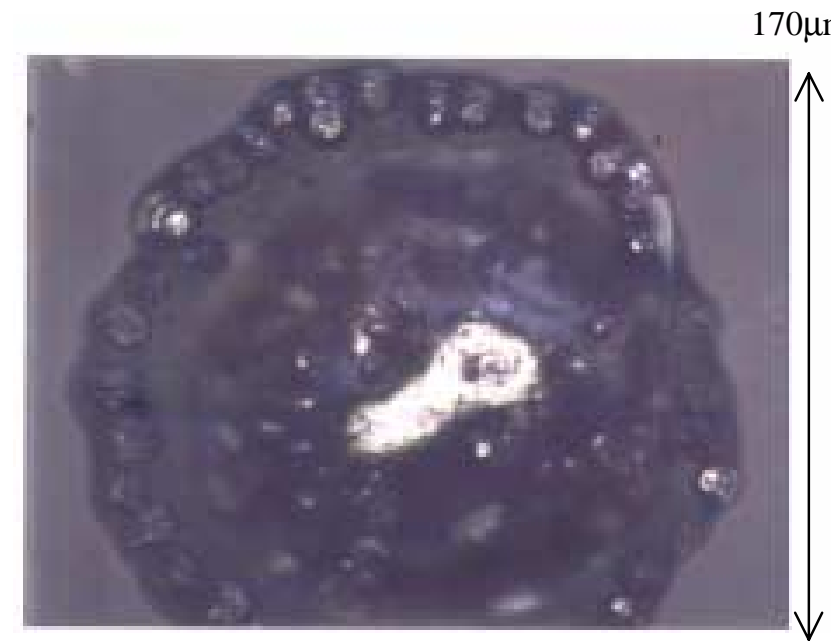

While the necks are forming in the outer layers, the inner layers contract rapidly into a molten pool which pulls in the outer solder particles and rapidly forms a single equilibrium shape. The early stages of neck expansion have been modelled by CFD and compared against observations of neck growth between $650 \mu \mathrm{m}$ diameter particles coated with flux and $20 \mu \mathrm{m}$ diameter particles in solder paste. In both 
cases the CFD model predicts neck growth at a more rapid rate than observed and this is attributed to the presence of an incompletely removed oxide layer on the particles. These predictions are described in greater detail in section 4 .

\section{Effects of atmosphere, aging and paste volume}

It has been shown that the very low levels of oxygen present in a nitrogen inerted reflow process has a very significant impact on the ability of small deposits of solder to coalesce. Experiments on small paste volumes of an RMA paste (volume $<0.4 \times 10^{-12} \mathrm{~m}^{3}$ ) resulted in incomplete coalescence of the particles. However when the reflow was conducted in an inert atmosphere, all deposits except the smallest $\left(<0.2 \times 10^{-12} \mathrm{~m}^{3}\right)$ reflowed without defect. The importance of oxygen diffusing from the atmosphere, and creating fresh oxide is also seen in surface analysis of planar solder surfaces heated in flux. Solder mass surfaces were examined using XPS to analyse the thickness and composition of oxide layers. The most likely interpretation of the data shows that the outer layers are a mixture of $\mathrm{SnO}$ and $\mathrm{SnO}_{2}$, in agreement with previous studies, [3] and the initial thickness of the layer was observed to be $\sim 2 \mathrm{~nm}$. After treatment in a weak rosin flux without activator, the oxide layer was found to have grown with increasing temperature. This is attributed either to the continued oxidation of the solder after heating had stopped but before analysis, or to the weakness of the flux, which was unable to compete with oxidation due to oxygen diffusing in from the surrounding atmosphere. Using a stronger flux did reduce the oxide layer thickness. These results will be reported in detail elsewhere.

Paste ageing can also have a significant effect on reflow capability. Incomplete reflow of a paste stored at $\sim 5^{\circ} \mathrm{C}$ for 3 months has been observed, while complete, defect-free reflow was observed with fresh paste of the same formulation. This is attributed to the action of fluxing agents on the surface of the solder particles during storage resulting in a reduced amount of active flux at reflow. Other explanations are also possible such as chemical reactions taking place between the flux constituents, or the production of a flux resistant layer at particle surfaces due to flux-metal reactions. It is not anticipated that the oxide layer would grow significantly due to diffusion at these temperatures and time-scales.

It has been observed that smaller volumes of paste do not reflow as rapidly or as well as larger volumes. Deposits from square $200 \mu \mathrm{m}$ apertures in a $150 \mu \mathrm{m}$ thick stencil reflowed well (paste volume $\sim 0.4 \times 10^{-12} \mathrm{~m}^{3}$ ) while deposits from $140 \mu \mathrm{m}$ apertures in the same stencil $\left(0.17 \times 10^{-12} \mathrm{~m}^{3}\right)$ exhibited solder balling. The most likely explanation of this phenomenon is the presence of a thinner layer of flux covering the particles as they reflow, as illustrated in fig.6. Similarly, smaller deposits are more likely to show partial particle coalescence. In the extreme case where a single particle is heated in air on a hot plate onto a $\mathrm{Cu}$ surface, no wetting of the $\mathrm{Cu}$ is observed. When the number of particles increases to 4-5 a very small amount of coalescence can be observed, but never full coalescence and reflow into a single solder mass.
Figure 6. Flux being expelled from inner layers during

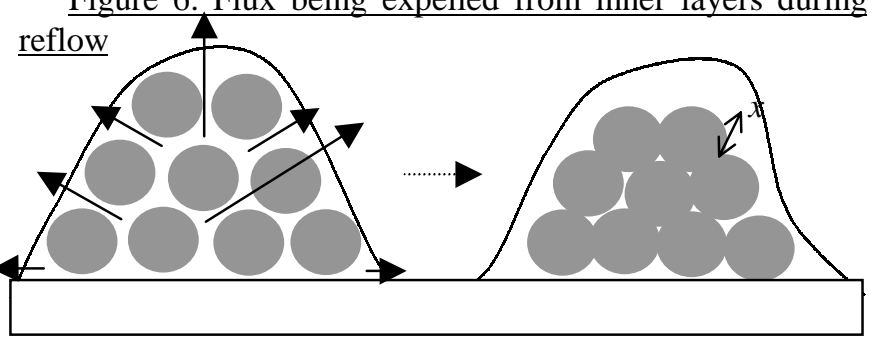

If gravity is neglected and it is assumed that the solder and flux form concentric hemispheres, then given a solder paste volume, $V$, and a volume fraction of particles, $f$, we might expect the thickness of the flux coating, $x$, to be given by approximately by:

$$
x=(3 V / 2 \pi)^{1 / 3}\left(1-f^{1 / 3}\right)
$$

Typically $f$ is expected to equal 0.75 once the volatiles have been driven off so that $x$ would be of the order of $0.07 \mathrm{~V}^{1 / 3}$. Taking the above examples, the expected flux layer is $5 \mu \mathrm{m}$ thick examples for the $200 \mu \mathrm{m}$ aperture deposit, while for the $140 \mu \mathrm{m}$ aperture, the flux layer is $4 \mu \mathrm{m}$ thick.

Surface tension will act quickly to thin down the flux layer if the wetting angle of the flux to the PCB is less than $90^{\circ}$. The time-scale for flux depletion is controlled by the ratio of surface tension to viscosity, while the final thickness of flux on the solder is given by the wetting angle. With a surface tension of $0.05 \mathrm{~N} / \mathrm{m}$ typical for polymers, a wetting angle of $30^{\circ}$ and viscosity of $100 \mathrm{~Pa} . \mathrm{s}$, CFD calculations predict a time scale of $2 \mathrm{~s}$ necessary to bring down a $5 \mu \mathrm{m}$ layer to $4 \mu \mathrm{m}$. Since solder coalescence and flux expulsion have a characteristic time of $\sim 1$ s, the extra flux volume expelled from larger deposits will help to shield the outer particles from oxygen during solder coalescence for longer. It should be noted that increasing the flux contact angle with the PCB could help reduce incomplete coalescence, but that this is not easy due to the high organic content of the flux keeping contact angles small. Experiments with low surface energy glass surfaces do not show significant reduction of flux spreading.

\section{CFD Simulations of coalescence without oxides}

The pattern of solder particle coalescence in the absence of any oxide layers is studied first. The simplest situation is that of two contacting spheres immersed in a viscous fluid (the vehicle; 'flux'), coalescing under the action of surface tension (see fig.7). In all of the following simulations the flux is treated as a Newtonian fluid. This situation can be used to test the accuracy of the CFD method.

Figure 7. Two particles coalescing; neck diameter shown

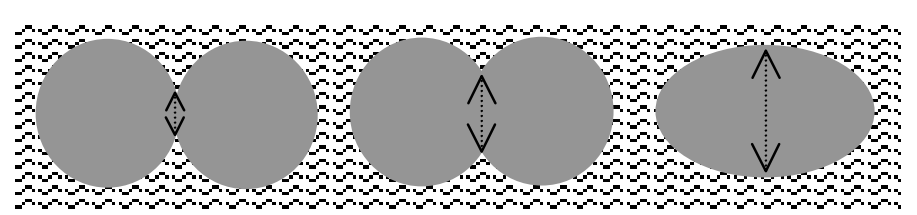


Two different numerical algorithms were used to compare against each other and against the experimental results. These were the level-set method [4] and the Volume of Fluid (VoF) method [5]. Both level-set and VoF use fixed meshes which do not distort throughout the simulation. The fluid interface is tracked by solving an additional set of equations for a scalar quantity that is advected with one of the fluids. The level-set method was implemented by enhancing the PHYSICA CFD code, while the VoF method is a standard option on the FIDAP code. An axisymmetric model could be used because of the rotational symmetry of the problem.

For two solder spheres coalescing, the time required for coalescence may be estimated on dimensional grounds. Firstly it is clear that the inertial effects, and hence fluid density, is unlikely to be relevant to the problem as we are in the low Reynolds number regime (this point has been explicitly verified in the CFD simulations). The remaining quantities; interfacial surface tension $(\sigma)$, flux viscosity $(\eta)$ ( $\eta$ is much greater than solder viscosity) and typical length scale $(l)$, can naturally be combined as:

$$
t=l \eta / \sigma
$$

to form a time constant that describes the process. Here we may use the particle radius, $a$, as the typical length scale so that eq.(2) becomes:-

$$
t=a \eta / \sigma
$$

Values for $\eta$ and $\sigma$ are estimated to be 100Pa.s and $0.4 \mathrm{~N} / \mathrm{m}$, from the literature. For 650 micron eq. 3 gives a time constant of $\sim 0.1 \mathrm{~s}$, while for 20 micron particles, eq.(3) predicts a time constant of $0.003 \mathrm{~s}$. Experimentally the growth of the interparticle necks was observed on video, and takes seconds rather than milliseconds for both types of particle, while the two CFD methods agree with each other and with the dimensional arguments (i.e. coalescence in milliseconds). Thus the CFD models do not take into account all of the relevant physical processes, and the most likely candidate for restricting particle coalescence is the partially disrupted oxide layers on the particles.

The CFD prediction for 20 micron spheres (coalescence in milliseconds) has been partially confirmed by video observation when isolated particles immersed in flux contact other particles or the central solder mass and coalesce in under 40ms (the time between one video frame and the next). These particles are thought to have had their oxide layers removed due to the large ratio of flux/solder present.

Figure 8 shows a comparison of the CFD methods with experiment. The viscosity of flux chosen in the CFD experiments was 800Pa.s for purposes of comparison, but even with this artificially high value, the experimentally observed neck growth is restricted by comparison. The two CFD methods do not agree very well early on in the simulation when the radius of curvature is very small. However the two methods do agree at later times when the curvatures involved are less severe.
Figure 8. Neck growth of $650 \mu \mathrm{m}$ spheres



The remaining CFD simulations described in this paper were carried out in FIDAP using the VoF method. The situation when an inner spherical pool of solder has formed, but the outer particles have not yet coalesced, is examined next. The problem can still be simulated using an axisymmetric geometry as shown in fig.9. The results show that a single sphere should merge with the pool (pool volume is 125 solder spheres), but that if there was an intermediate sphere, then a proportion of the solder would remain isolated from the main mass. This is illustrated in fig. 9 where three spheres are shown in various degrees of coalescence. If instead of three outer spheres, we had only two then the isolated remnant would be only $10 \%$ of an original particle mass.

\section{Figure 9. Coalescence of outer spheres}

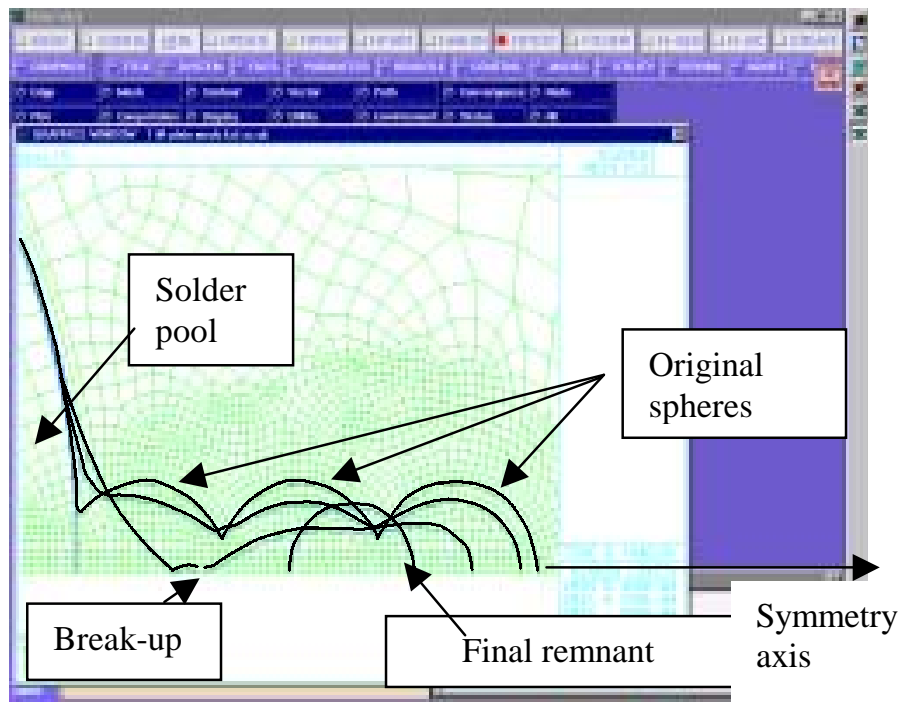

These results would tend to indicate that the outer particles would leave behind solder balls around the main deposit in the case of rapid coalescence of oxide free surfaces. This has not been observed in the video observations on real, oxide constrained particles. A 2-D model of particle coalescence is shown in fig.10, without an initial central solder mass present. 
Figure 10. 2-D model of coalescing cylinders

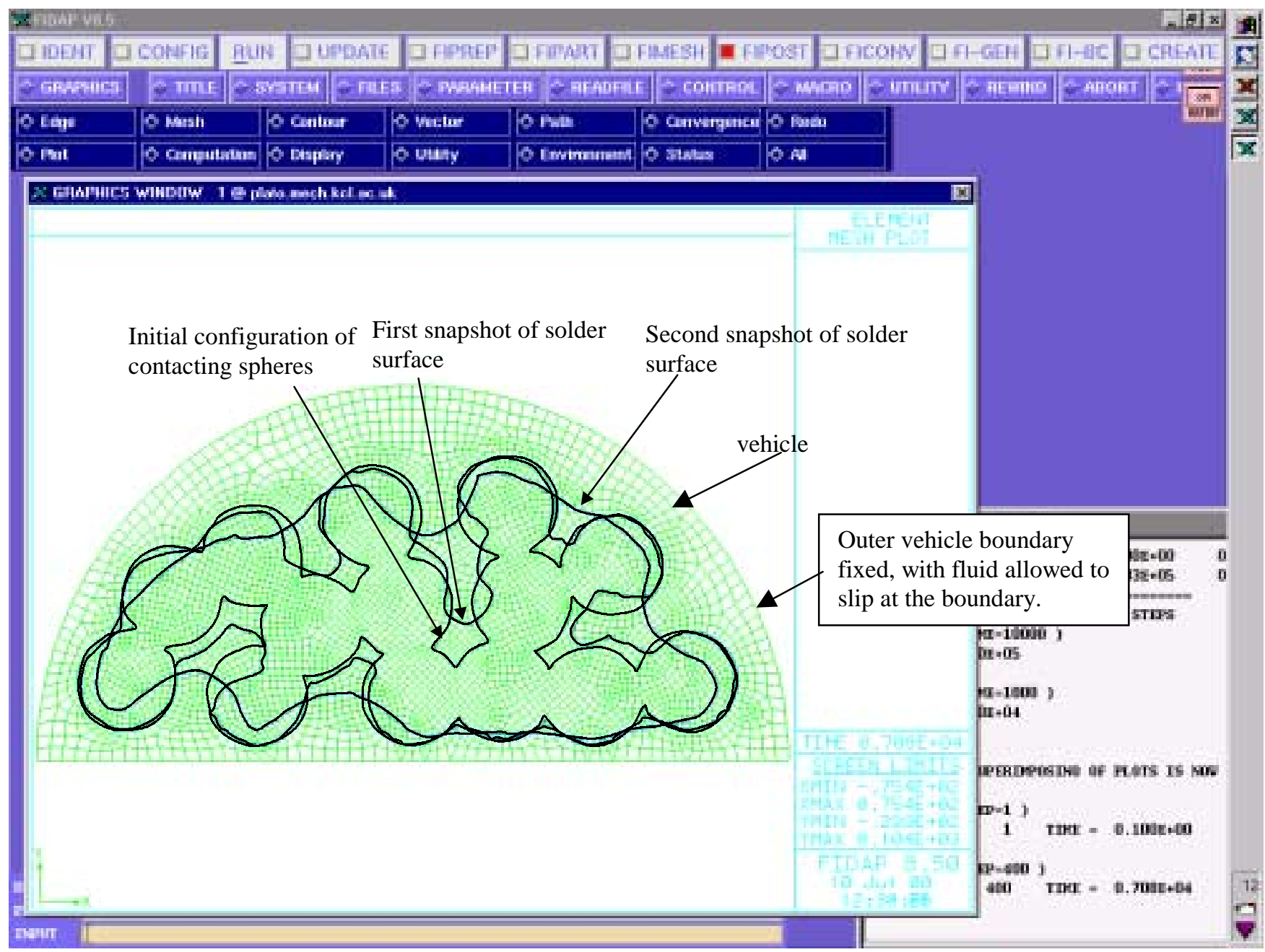

This figure indicates that no particles would be left behind as the particles coalesce. However it is inappropriate to compare this situation with that of fig. 9 because replacing the spheres in fig.9 with cylinders (i.e. performing a 2-D calculation rather than an axisymmetric one) would also result in a single solder mass without any remnants. The solution would be to perform a fully 3-D calculation, but at present even a simulation of just three spheres coalescing is problematic in terms of the computer power required.

\section{Modelling the effect of Oxides}

In a first step towards a full model of paste coalescence taking into account oxide formation and reduction on the surface of the solder particles, a simplified 2-D model has been constructed. The coalescence of oxide free solder cylinders has been coupled to a scenario where the presence of oxygen diffusing from the atmosphere, through the flux and into the solder locally freezes the solder. This is implemented by letting the solder viscosity (normally negligible) rise to a value 100 times greater than that of the flux when an arbitrary critical threshold is reached.
The results are shown in figs.11-13, where both the solder surface and the critical oxygen level is shown. Figure 14 shows the equivalent solder surface reached in the absence of oxygen.

\section{Figure 11. Initial Configuration and mesh}

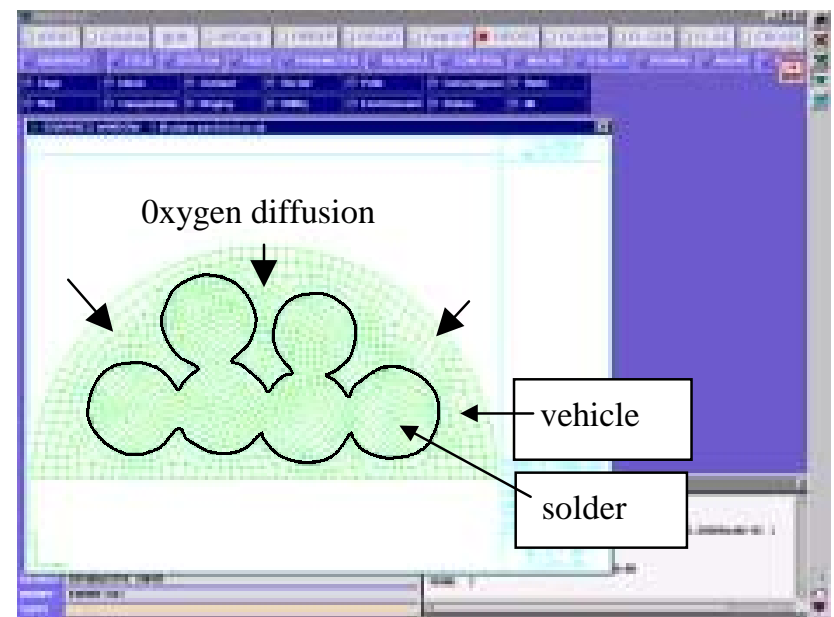


Figure 12. Critical Oxygen level reaches outer particle

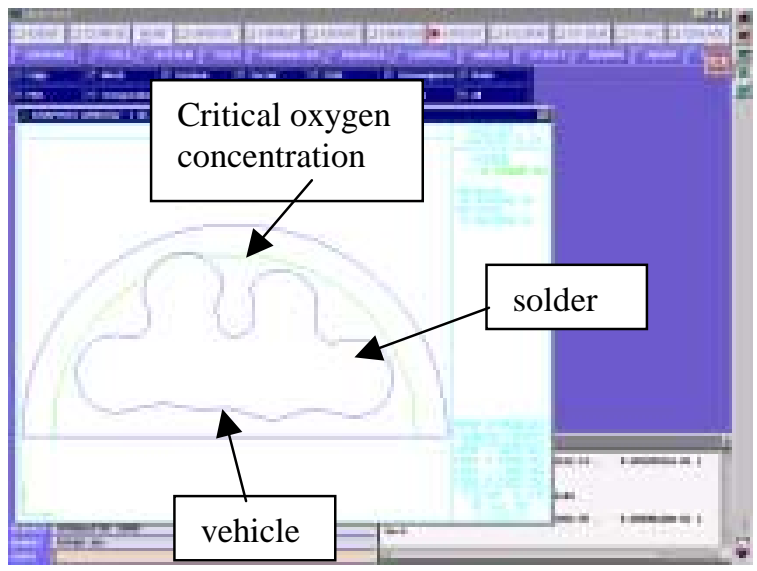

Figure 13. Outer particle incompletely coalesced at end of simulation

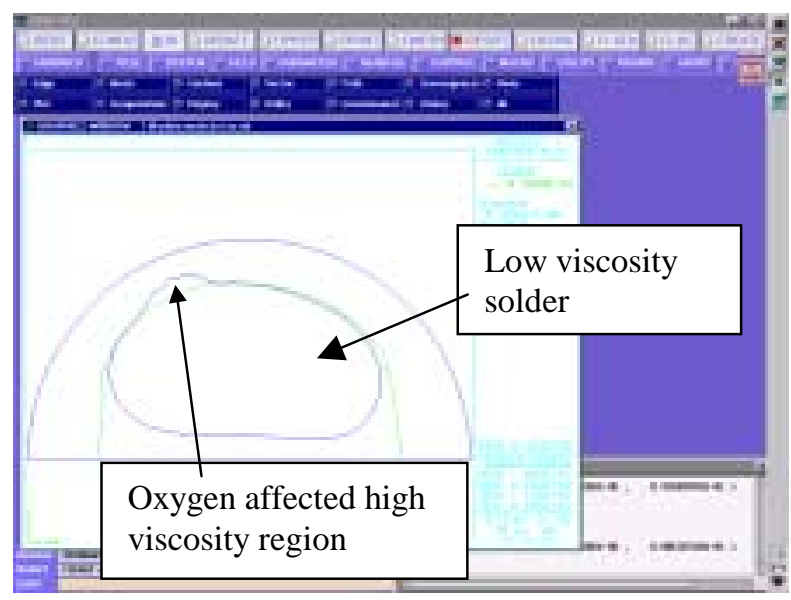

Figure 14. Time-step of fig.13 without oxide effect

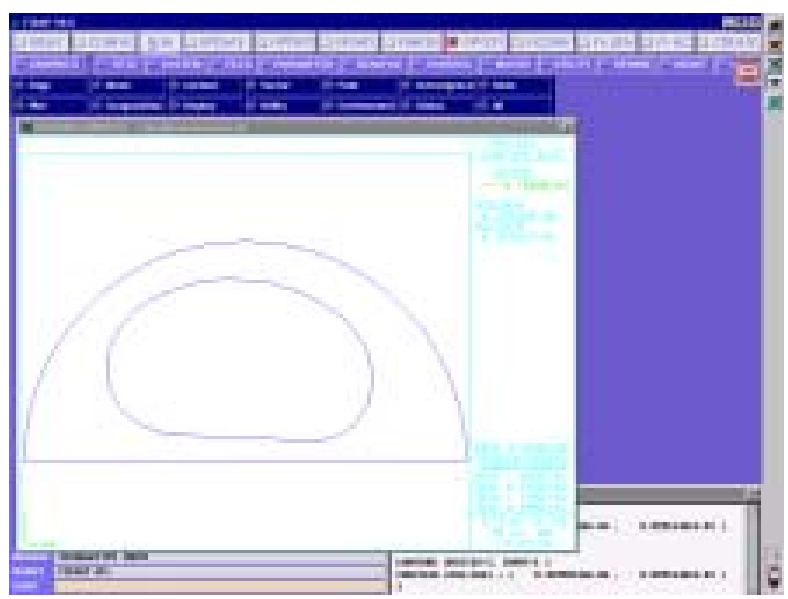

Figure 13 closely resembles solder deposits that have been observed with incompletely coalesced particles. However, the effect of the oxide (a thin skin on the solder surface) is represented in these models as a bulk solder effect.

The model can be improved by adding an initial oxide layer and chemical reactions. The chemicals present are metal $\{1\}$, oxygen $\{2\}$, oxide $\{3\}$, flux $\{4\}$, and a flux oxide reaction product $\{5\}$. The reactions are set as:

$$
\{1\}+\{2\} \rightarrow\{3\} \text { rate constant } k_{1}
$$

and

$$
\{3\}+\{4\} \longrightarrow\{5\} \text { rate constant } k_{2}
$$

As well as the reaction rates $k_{1}$ and $k_{2}$, the diffusion rates and concentrations of oxygen and flux in the vehicle, also control the process. The initial distribution of chemical species is shown in fig. 15 .

$\underline{\text { Figure 15. Initial Geometry and chemical distribution }}$

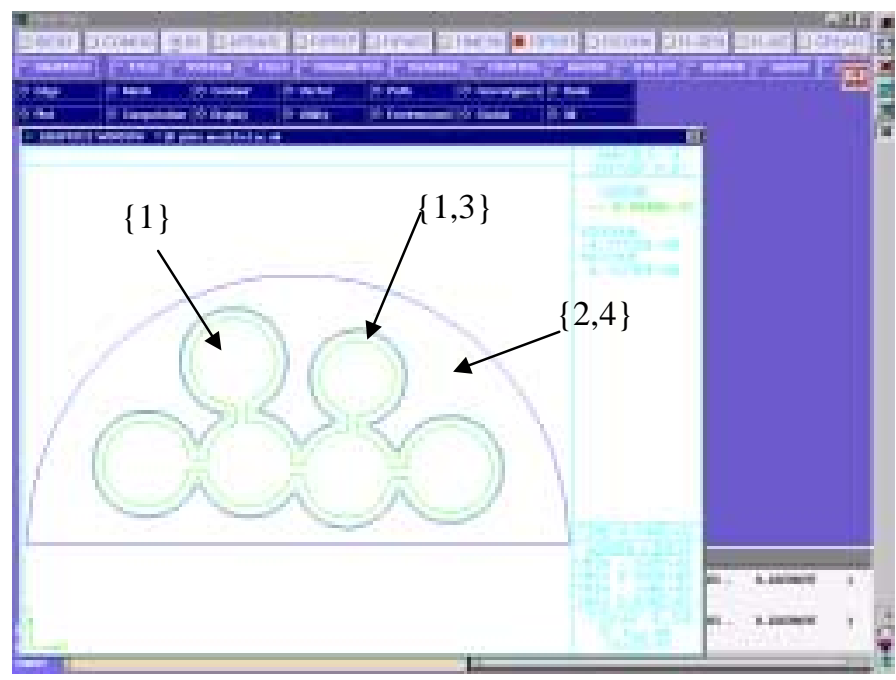

Species $\{5\}$ is not initially present. The oxide inhibits solder movement, again by increasing the local viscosity when it is present at sufficient concentration. Note that the oxide layer is relatively thick $(\sim 2 \mu \mathrm{m})$ to allow for the coarseness of the mesh; the oxide layer is initially 3 elements thick as shown in fig. 16 .

$\underline{\text { Figure } 16 \mathrm{Mesh} \text { and oxide layer }}$

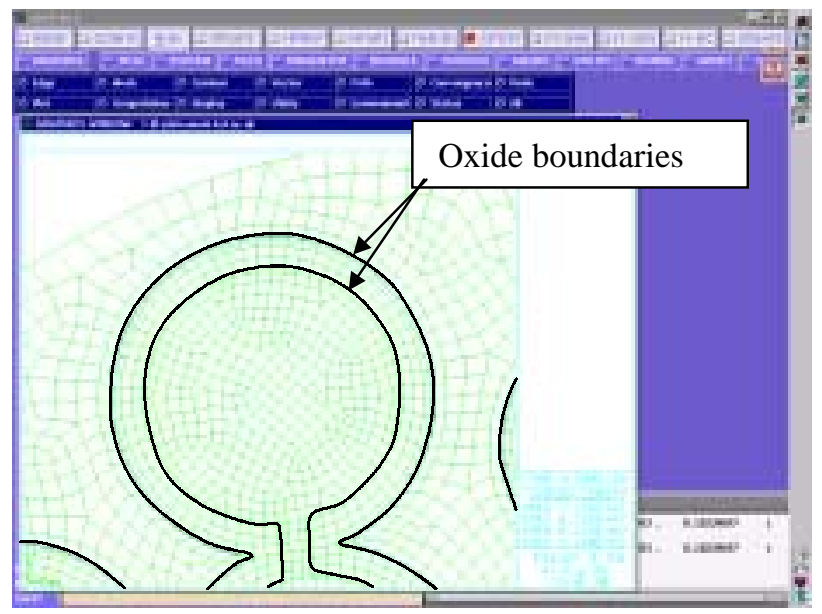


Figs.17-18 show intermediate stages of coalescence, showing the break-up of the oxide layer. Oxygen is diffusing in from the outer boundary as before.

Figure 17 Oxide layer thinned down and start of break up

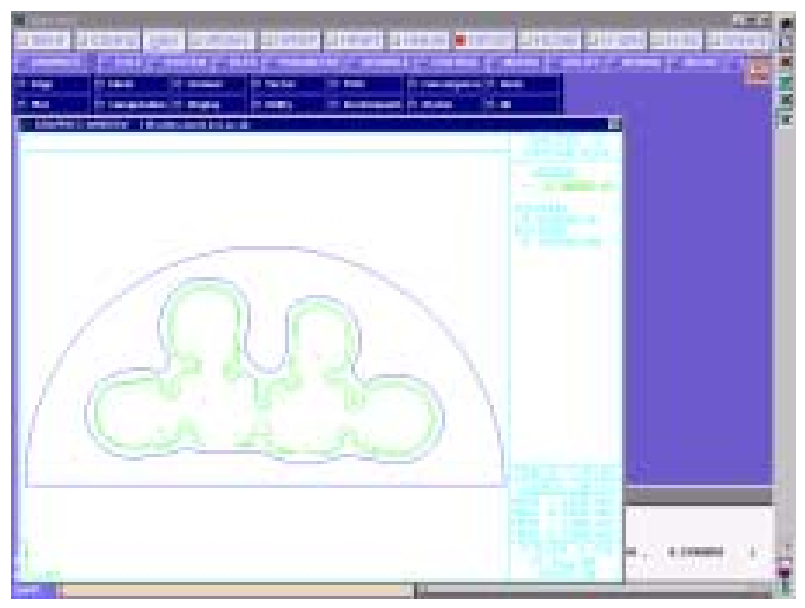

Figure 18. Remnants of oxide layer

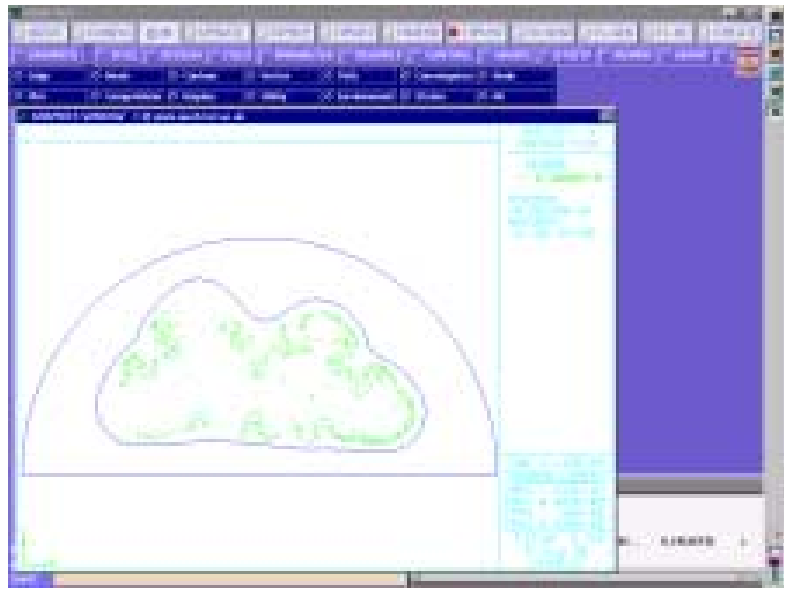

On real solder particles, the oxide layer is only nanometers thick, rather than microns as simulated here. Similarly, it is a solid layer and will exhibit elasticity whereas here the oxide layer is modelled as an extremely viscous fluid, slowing down its from deformation, and even this becomes problematic as the oxide layer thins down in the simulation because of difficulties in numerical convergence. Nevertheless figs.17-18 do show the patchy nature of the oxide as it breaks up and allows coalescence to progress.

\section{Conclusions}

The reflow experiments, video observations and CFD simulations have shown that the oxide layer, and in particular the rate of reoxidation of the solder surface, play a key role in achieving defect free soldering. Applying heat through the substrate is not as effective as convection type heating, and for small paste volumes, a reduced oxygen concentration atmosphere is essential. It has been postulated that the reason smaller volumes of paste reflow poorly is the smaller reservoir of flux held between the particles which must come to the surface as the particles coalesce. The pattern of particle colaescence; formation of necks, followed by membrane - like structures between particles, followed by rapid coalescence, has been described.

Future work will explore the parameter space of diffusion rates and reaction rates, with experimentally determined values for these constants allowing quantitative predictions to be made. The work also needs to be extended to 3-D. since it has been shown that there are significant differences in coalescence patterns between 2-D and 3-D simulations. Furthermore, more sophisticated models of the oxide-vehicle interface need to be developed. The nature of the oxide layer separating particles just before reflow; its thickness and extent of surface coverage is being studied by electrical means, and may be used in future to supply the initial conditions for the oxide in CFD simulations. [6]

\section{Acknowledgments}

This work was conducted under UK EPSRC grants GR/L61767 and AF/990541, and with the help of an industrial and academic consortium of partners including Multicore Solders Ltd., Celestica Europe Ltd., Mitel Semiconductor, DEK printing machines, Matra Dynamics Intarsia Corp. and Salford University. Particular thanks to Tennyson Nguty (Salford) and Mike Hendrikson (Celestica) for the work on solder paste volume and nitrogen reflow.

\section{References}

1. Conway, Paul P., et al, "Experiments to Improve the Understanding of Process Defects Such as Partial Reflow and Solder Balling as Occurring in the Soldering of Solder Paste Materials", Proc. Productronica '95, Munich, 1995, pp 53-60.

2. Natishan, M E, et al, "In Situ Study of Solder Paste behaviour during reflow using the Hot-Stage Environmental Scanning Electron Microscope," J. Electronics Manufacturing, Vol. 7, No. 1, (1997), pp.29-40.

3. Kuhmann, J F, et al, "Oxidation and Reduction of Liquid $\mathrm{SnPb}$ (60/40) under Ambient and Vacuum Conditions," J. Electrochem. Soc., Vol. 145 (1998), pp.2138-2142.

4. Wheeler, D, et al, "Modelling the Melting and Solidification of Solder Material," Advances in Electronic Packaging, ASME, EEP- Vol. 26-2 (1999), p 397-404.

5. Hirt, C, et al, "Volume of Fluid ( $\mathrm{VoF})$ method for the dynamics of free boundaries," J. Comp. Physics, Vol. 39, No. 1 (1981), pp.201-225

6. Mannan, Samjid H., et al, "Observations of Solder Paste Reflow by means of Electrical Measurements", To be published in Proc. International Symposium on Electronic Materials And Packaging 2000 (Hong Kong). 